\title{
Numerical study on OWC devices integrated into a floating breakwater
}

\author{
Madjid Ghodsi Hassanabad', Mojtaba Shegeft ${ }^{2}$ \\ ${ }^{1}$ Department of Marine Science and Technology, Tehran Science and Research Branch, Islamic Azad University \\ Tehran, Iran. \\ M.ghodsi@srbiau.ac.ir; Mojtaba.shegeft@gmail.com
}

\begin{abstract}
This paper describes a numerical investigation of hydrodynamic performance of oscillating water column (OWC) as a wave energy converter integrated into a floating breakwater using 3D and 2D computational fluid dynamics (CFD) modelling. The numerical results of 3D model of OWC chamber with parabolic side walls were validated. In order to do that, the power of air passing through the turbine in the model was compared with results of other researches, and the results show a good agreement. Then, the usability of OWC devices as floating breakwater were investigated by 2D numerical models to do that 3 types of OWC models analysed and finally the best model is selected due to the damping of the waves and performance. To create a free surface in the CFD program, the domain must be divided into two phases of a fluid. Therefore Volume of Fraction (VOF) formulation have been used in the models. The continuity and momentum equations were selected as the governing equations.
\end{abstract}

Keywords: Oscillating water column (OWC), Wave attenuation, Floating breakwater.

\section{Introduction}

Renewable energy is an urgent matter in the world. Due to the reduction of fossil fuels and many irreversible harms they may cause, investigation in this industry and finding alternative sources of energy can be convenient. Wave energy could be a good fit. In order for this sources of energy to be useful, it needs to be captured and transformed into useful energy such as electricity. Among the different techniques used for wave energy converters, the Oscillating Water Column (OWC) devices stand as one of the most promising ones. In addition, its inherent simplicity is presumably the greatest advantage of OWC as a wave energy convector device which can generate sustainable energy, therefore using OWC devices can be convincing in many countries.

In order to make OWC device more Cost-effective in production and use, compared to other form of wave energy converters, using OWC devices with other offshore or coastal structures such as breakwaters is suggested. This way, the wave energy can be attenuated to maintain costal structures in harbour zones, and in the same time energy can be gained from the ocean so the costs of producing OWC devices will be reduced through integrate solutions, simple manufacture and ease of installation to facilitate a competitive cost of energy in commercial.

One of the goals of the present project is to promote the use of this device by increase the hydrodynamic performance of OWC devices integrated into a breakwater. So further investigation such as numerical (CFD) modelling need to be done to consider all challenges such as convert wave energy into a useful energy form, adaptability to a wide variety of different locations and ocean condition, survivability in the ocean over time and in storm condition. Due to the lack of fully understanding the OWC device, further studies are needed. According to the specific area.

The Structure of OWC devices consists of a large chamber, one end is open to the sea, and the other leads into a orifice, when a wave approaches the device water is forced into the chamber, applying pressure on the air, already trapped inside the structure. As a result of this huge pressure the air escapes to atmosphere through the turbine on the other side of orifice, thereby producing electricity. When the wave retreats and the water surface level falls, the air from the outside the orifice is sucked back in to the chamber. As the water surface level inside the chamber moves up and down, it compresses and decompresses the air, respectively.as a result, electricity continues to be generated.

Evance [1] studied OWC analytical model as a pair of parallel vertical plates, based on linear water wave theory. Then the wave power absorption by systems of oscillating surface pressure distributions was developed by Evance [2]. Many researchers have focused on hydro hydrodynamic analysis of OWC power plant (eg, Thiruvenkatasamy et al. [3], Josset and Clement [4], João C Henriques et al. [5]). McKinley et al. [6] studied the development of a Pseudo-3D testing technique for 
the prediction of extreme loads on an oscillating wave surge converter. Their investigations show that the hydrodynamics and magnitudes of slams experienced by device in a $2 \mathrm{D}$ environment vary significantly from those experienced in the more realistic 3D environment. Elhanafi [7] investigated the prediction of regular wave load on a fixed offshore OWC using CFD. Then, he continued his studies on Experimental and numerical model [8]. Due to the lack of fully understanding the OWC device, further studies are needed either experimentally, numerically, or a combination of these methods.

\section{Governing equations}

It is assumed that the fluid is incompressible. The VOF model can model two or more immiscible fluids by solving a single set of momentum equations and tracking the volume fraction of each of the fluids throughout the domain for the $\mathrm{q}^{\text {th }}$ phase, this equation has the following form:

$$
\begin{gathered}
\frac{1}{\rho_{\mathrm{q}}}\left[\frac{\partial}{\partial_{\mathrm{t}}}\left(\mathrm{a}_{\mathrm{q}} \rho_{\mathrm{q}}\right)+\nabla \cdot\left(\mathrm{a}_{\mathrm{q}} \rho_{\mathrm{q}} \overrightarrow{v_{\mathrm{q}}}\right)=\mathrm{S}_{\mathrm{a}_{\mathrm{q}}}+\sum_{\mathrm{p}=1}^{\mathrm{n}}\left(\mathrm{m}_{\mathrm{pq}}^{\cdot}-\mathrm{m}_{\mathrm{qp}}\right)\right] \\
\sum_{\mathrm{q}=1}^{\mathrm{n}} \mathrm{a}_{\mathrm{q}}=1
\end{gathered}
$$

Where $\mathrm{m}_{\mathrm{qp}}$ the mass transfer from phase $\mathrm{q}$ to phase $\mathrm{p}$ is, $\mathrm{m}_{\mathrm{pq}}$ is the mass transfer from phase $\mathrm{p}$ to phase $\mathrm{q}$. The source term $\mathrm{S}_{\mathrm{a}_{\mathrm{q}}}$ is zero.

In the single fluid model, the phase function $\mathrm{a}_{\mathrm{q}}$ is equal to 1 in water and 0 in air. The interface is defined by $\mathrm{a}_{\mathrm{q}}=0.5$. The magnitude of the physical characteristics of the fluids depends on the local phase. They are defined as:

$$
\begin{aligned}
& \rho=\rho_{1} \text { And } \mu=\mu_{1} \text { if } \mathrm{a}_{\mathrm{q}} \geq 0.5 \\
& \rho=\rho_{0} \text { And } \mu=\mu_{0} \text { if } \mathrm{a}_{\mathrm{q}}<0.5
\end{aligned}
$$

Where $\rho_{0}, \rho_{1}, \mu_{0}$ and $\mu_{1}$ are the densities and viscosities of fluid 0 and 1 , respectively [9].

The mixture of air and water is considered as a fluid of variable density. The dynamic viscosity coefficient of the fluid are defined as:

$$
\begin{aligned}
& \rho=\alpha_{0} \rho_{0}+\alpha_{1} \rho_{1} \\
& \mu=\alpha_{0} \mu_{0}+\alpha_{1} \mu_{1}
\end{aligned}
$$

Where $\alpha_{0}$ and $\alpha_{1}$ are voulume fraction of air and water respectively.

The equation was solved for hydrodynamic analysis was potential equation. The boundary conditions were solid wall B.C., kinematic and dynamic free surface B.C., far field B.C. (Sommerfeld), and symmetry B.C. [10, 11]. BEM was selected as a numerical method for its simplicity in mesh generation and rapid processing in PDE solving. It assumed that boundary conditions are nonlinear and finite difference method was used for discretization of boundary conditions. Also, solving domain divided into 3 zones for preventing singularities (zoning method).

Another part of the problem is the modeling of the air volume enclosed in the OWC chamber above the inner free surface. The air pressure $\mathrm{P}(\mathrm{t})$ in the chamber is a new variable for which a new equation is necessary. This pressure reacts on the hydrodynamic problem through the dynamical free-surface condition. Hydrodynamics and aerodynamics are therefore strongly coupled. Figure. 1 shows a schematic view of OWC chamber with trapped air parameters on it. 


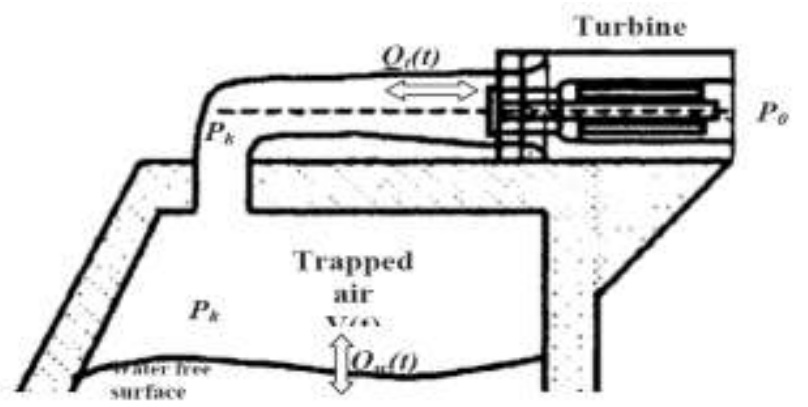

Fig. 1: Scheme of OWC chamber.

Table 1: Parameter's definition.

\begin{tabular}{|c|c|}
\hline Parameter & definition \\
\hline $\mathrm{g}$ & gravitational acceleration \\
\hline$c_{v}$ & specific heat for constant volume \\
\hline$c_{p}$ & specific heat for constant pressure \\
\hline$P(\mathrm{t})$ & relative pressure of the trapped air \\
\hline$P_{0}$ & atmosphere pressure \\
\hline$K_{t}$ & Turbine Drop Coefficient \\
\hline $\mathrm{t}$ & time \\
\hline$V(t)$ & Trapped air column volume \\
\hline$\varepsilon$ & capture width \\
\hline$\gamma$ & adiabatic coefficient \\
\hline
\end{tabular}

In order to calculating the power-take-off device influence, the characteristic relation of turbine is needed and in this investigation it is linear between the air flow and the pressure drop across the turbine:

$$
Q_{t}(t)=-\frac{P(t)-P_{0}}{K_{t}}=-\frac{P_{k}(t)}{K_{t}}
$$

The definition of the air flow $Q_{t}(t)$ through the turbine, notice that it is counted positively when it comes into the plant and negatively when it goes out $\left(Q_{t}(t)<0 \rightarrow \varepsilon=0, Q_{t}(t)>0 \rightarrow \varepsilon=1\right)$

The final form of pressure equation is as follows:

$$
\frac{\dot{P}_{k}(t)}{P_{k}(t)+P_{0}}=\gamma\left[-\frac{P_{k}(t)}{K_{t} V(t)}\left(1-\varepsilon \frac{\left(P_{k}(t)+P_{0}\right)^{1 / \gamma}-P_{0}^{1 / \gamma}}{\left(P_{k}(t)+P_{0}\right)^{1 / \gamma}}\right)+\frac{Q_{w}^{k}(t)}{V^{k-1}(t)-\Delta t Q_{w}^{k}(t)}\right]
$$

$\gamma=\left(c_{p} / c_{v}\right)$ which is considered to be 1.4 for air.

The dimensionless and discrete shape of this equation is as follows.

$$
\begin{aligned}
& \hat{P}_{k}^{k}(t)=\hat{P}_{k}^{k-1}(t)+\gamma \Delta \hat{t}\left(\hat{P}_{k}^{k-1}(t)+\hat{P}_{0}\right) \\
& {\left[-\frac{\hat{P}_{k}^{k-1}(t)}{\hat{K}_{t}\left(\hat{V}^{k-1}(t)-\Delta \hat{t} \hat{Q}_{w}^{k}(t)\right)}\left(1-\varepsilon \frac{\left(\hat{P}_{k}^{k-1}(t)+P_{0}\right)^{1 / \gamma}-P_{0}^{1 / \gamma}}{\left(\hat{P}_{k}^{k-1}(t)+P_{0}\right)^{1 / \gamma}}\right)+\frac{Q_{w}^{k}(t)}{V^{k-1}(t)-\Delta t Q_{w}^{k}(t)}\right]}
\end{aligned}
$$

In this regard, new dimensionless parameters are introduced which are defined as follows. 


$$
\hat{P}=\frac{P}{\rho g L} \quad ; \quad \hat{Q}=\frac{Q}{L^{2} \sqrt{L g}} \quad ; \quad \hat{V}=\frac{V}{L^{3}} \quad ; \quad \hat{K}_{t}=\frac{K_{t}}{\rho} \sqrt{\frac{L^{3}}{g}}
$$

In which $\rho$ is the density of the sea water.

Table 2: The details of the input wave and geometric details of the OWC power plant based on Fig. 2.

\begin{tabular}{|c|c|c|c|c|}
\hline Amounts & Dimensionless parameters & definition & Amounts & Parameters \\
\hline 0.0139 & $\hat{A}$ & Input wave domain & 0.475 & $A(m)$ \\
\hline 2.517 & $\hat{T}$ & Incoming wave frequency period & 4.7 & $T(\mathrm{sec})$ \\
\hline 0.439 & $\hat{h}$ & depth of water & 15 & $h(m)$ \\
\hline 1 & $\hat{L}$ & Input wavelength & 34.2 & $L(m)$ \\
\hline 0.537 & $\Delta \hat{t}$ & Time step & 0.5 & $\Delta t(\mathrm{sec})$ \\
\hline 2.193 & $\hat{X}_{\text {front }}$ & Front distance & 75 & $X_{\text {front }}(m)$ \\
\hline 0.585 & $\hat{X}_{\text {behind }}$ & The back wall of OWC to the shore & 20 & $X_{\text {behind }}(m)$ \\
\hline 0.292 & $\hat{X}_{f s}$ & Free surface length inside the chamber & 10 & $X_{f s}(m)$ \\
\hline 1.8625 & $\hat{K}_{t}$ & Turbine Drop Coefficient & 119.4 & $K_{t}$ \\
\hline
\end{tabular}

OWC with parabolic side walls was investigated in 3D study as shown in Figure. 2. The problem of this study divided into two parts and one of these parts was solved due to symmetry. Figure. 2 shows the geometry of the problem.
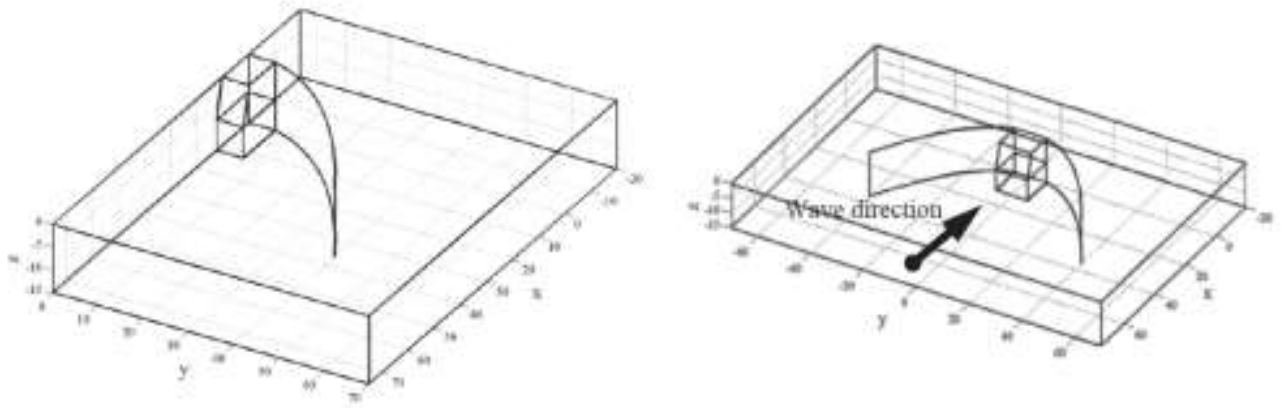

Fig. 2: Geometry of the problem.

The mathematical formula for parabolic shape is:

$$
x=\frac{-2 x_{\text {facus }}+\sqrt{4 x_{\text {facus }}^{2}+b^{2}}}{b^{2}} y^{2}+\frac{2 x_{\text {facus }}-\sqrt{4 x_{\text {facus }}^{2}+b^{2}}}{4}
$$

Where $\mathrm{x}$ and $\mathrm{y}$ are coordinate axes according to Figure. 2 , $\mathrm{x}$ focus is focus of the parabola which is $10 \mathrm{~m}$ and $\mathrm{b}$ is the width of power plant which is $12 \mathrm{~m}$.

\section{3D NUMERICAL MODELLING of OWC}

In this section 3D numerical modelling of OWC chamber with parabolic side walls was investigated. Quadrilateral meshes generated on boundaries of three zones. Figure. 3 shows meshes on the geometry of solution domain and three zones close to each other. 

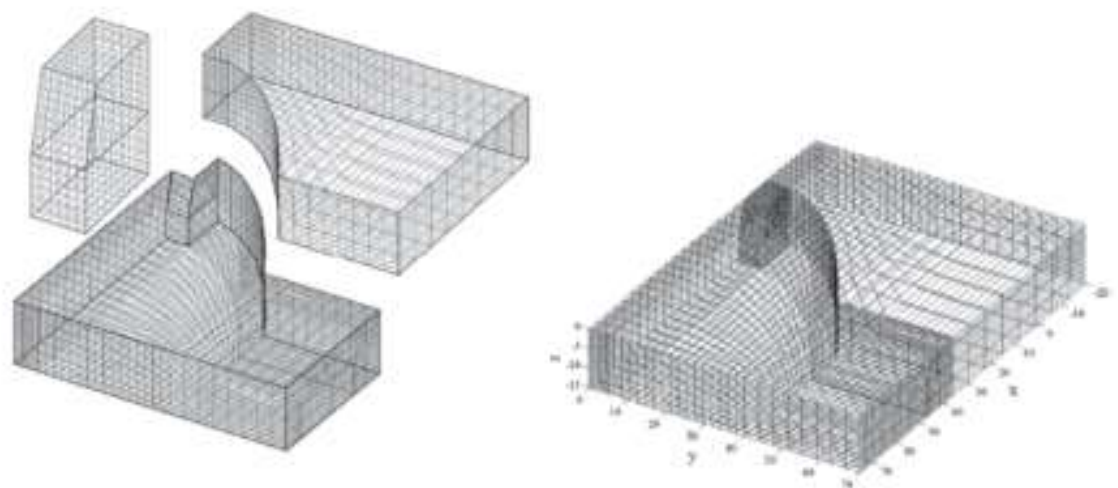

Fig. 3: Quadrilateral meshes on surfaces of three zones and quadrilateral meshes on whole of the solution domain.

For nearly singular points in BEM, analytical integration was used instead of Gauss integration. This caused convergence in time marching [12]. The results of this study were compared with Josset and Clement research. Figure. 4 shows the comparison between present study and mentioned research for a specified OWC chamber. Also, capture width parameter $\varepsilon$ $=P_{m t} / P_{m i}$ was compared with Josset and Clement results. In this formulation, $P_{m t}$ is average pneumatic power and $P_{m i}$ is mean incident power per meter of wave crest. Figure. 4 shows this comparison.

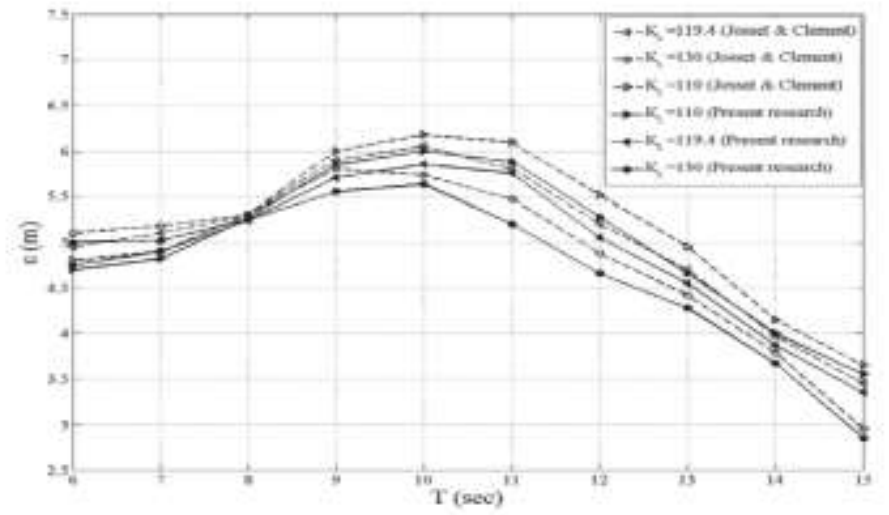

Fig. 4: Capture width of Pico Plant OWC versus wave period (wave amplitude=0.78m).

The overall shape of the diagrams in Figure 4 is similar to each other but the calculated results in the present study is slightly lower than the results of the Josset and Clement research, This can be due to the slight difference in the geometry of the two power plants in the two studies. The amount of absorbed air power of OWC is shown in a comparison of two studies using the following equation in Figure. 5

$$
P_{m i}=\frac{\rho g^{2}}{8 \pi} A^{2} T
$$

In which mean incident power is $P_{m i}(\mathrm{kw})$ incident wave period is T (s) and incident wave amplitude is A (m). 


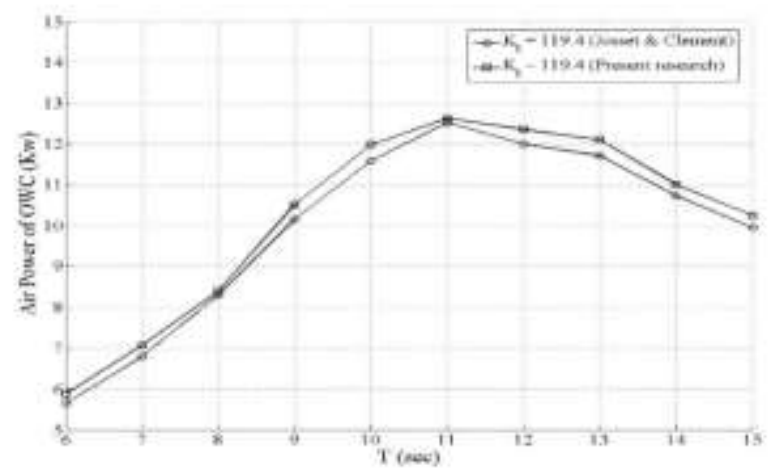

Fig. 5: The power of air passing through the turbine in different periods in kilowatts.

The considered 3D BEM model was previously validated with Josset and Clement research. The results shown in Figures. 4 and 5 illustrate a good accordance between these two studies. For more information, refer to the article provided by the author [13].

\section{2D numerical modelling:}

In 2D numerical wave tank, the top of the model (AC) opening of the tank was modelled as a pressure inlet boundary, with the pressure set to a gauge pressure of zero. The bottom (BD) was modelled as a no-slip wall. The inlet, (AB) or Flap type wave generator, was simple a velocity inlet that made use of the open cannel wave boundary function in Fluent. The boundary (CD) was modelled as pressure outlet that make use of open channel boundary function, to reduce the unwanted reflected waves from outlet boundary, the numerical beach function was used in the model.

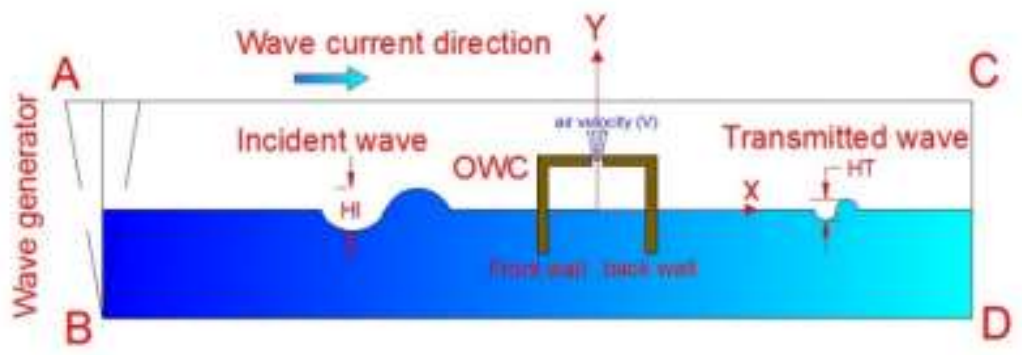

Fig. 6: The sketch of the OWC device and wave tank.

In this section, performance of 3 types of OWC with different dimensions as a wave energy converter and breakwater was studied numerically. Finally, the best model was selected. During the investigation wavelength (L) was constant (1.5 $\mathrm{m})$. According to different incident wave heights $\left(\mathrm{H}_{\mathrm{I}}\right), 0.1,0.14,0.18,0.22$, models were examined. The Depth of water was $1.35 \mathrm{~m}$ during the numerical study.

In the first and the second model the length of the back and front wall of OWC was considered the same size but their levels from the water free surface were different, the third model is a combination of these 2 types of model. The role of meshing is so important in CFD simulation. Quadrilateral elements are used in the model. To make sure that the solution is independent of mesh resolution, two types of model with different mesh size compered to each other, fist one with 25348 cells and second one with 6292 cells. The results value of mesh1 was within acceptable value of the results of the mesh2 and the solution was independent of the mesh. To reduce the simulation run time mesh1 is used in the 2D numerical simulation.

Due to improving the quality of the mesh design and reducing the time steps, mesh dimensions around the OWC are 
smaller than the other areas. Total cells are counted 6292 faces and nodes count 12799 and 6506 respectively. The sketch of these 3 types of model and mesh design can be seen in the figures 7,8 .

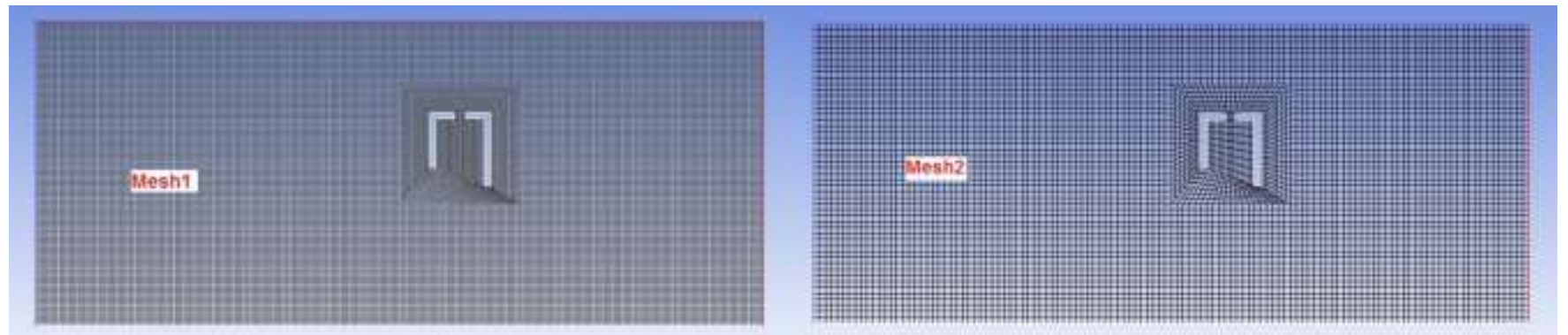

Fig. 7: mesh design.

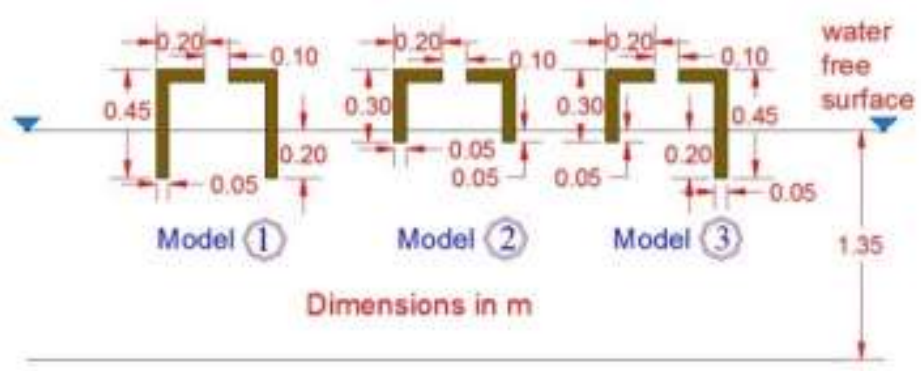

Fig. 8: The sketch of 3 types of models.

Figure. 9 shows snapshots of the water surface profile and different phases in the wave tank. In this figure, to create a free surface in the CFD program, the domain must somehow be divided into two phases of a fluid. Fluent can model the effects of open-channel flow using a Volume of Fraction (VOF) formulation, the free water surface configuration is defined as the VOF function, $\mathrm{F}=0.5$. The wave transformations around the OWC and the wave attenuation can be carefully investigated using figure 9 and figure 10 shows velocity vectors and its magnitude around the OWC.

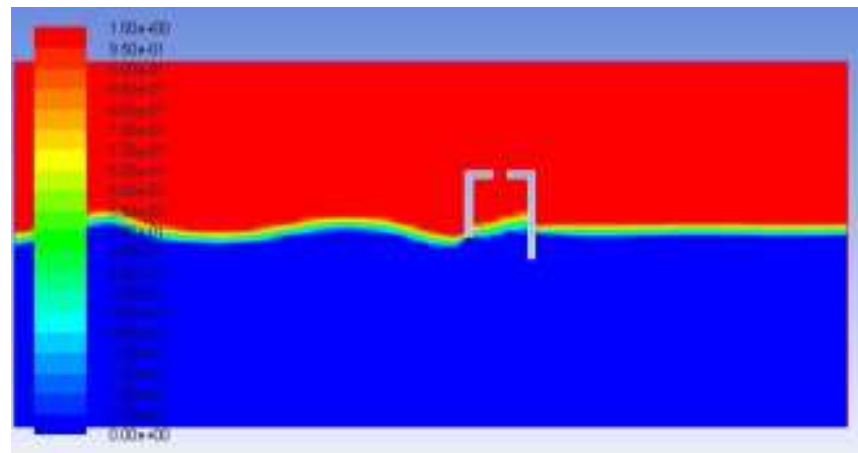

sinken

Fig. 9: Wave attenuation in numerical study.

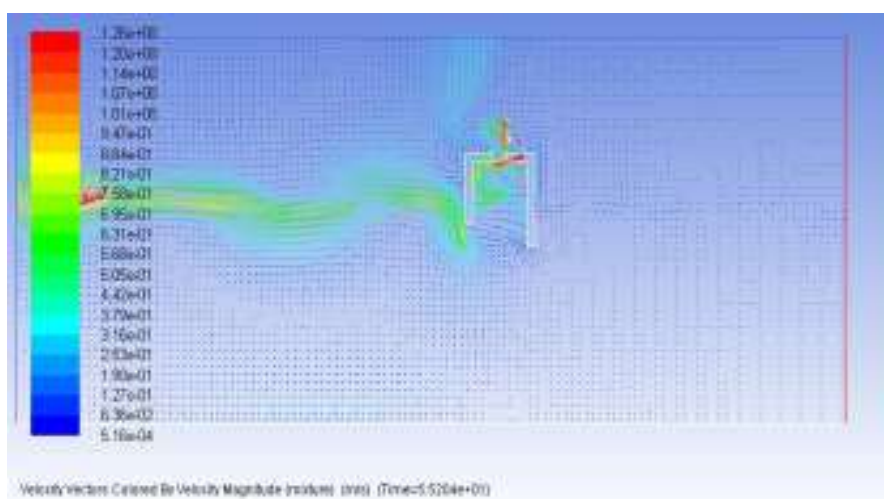

Fig. 10: velocity vectors around floating OWC.

Wave attenuation is calculated by equation $\left(1-\frac{\mathrm{H}_{T}}{\mathrm{H}_{\mathrm{I}}}\right)$. In this equation the transmitted wave height is defined as $\left(\mathrm{H}_{\mathrm{T}}\right)$ and the incident wave height is defined as $\left(\mathrm{H}_{\mathrm{I}}\right)$. In the table $3(\mathrm{~V})$ is air velocity from the orifice. 
Table 3. Results extracted from the 2D numerical models.

\begin{tabular}{|c|c|cc|c|c|}
\hline Model & $\mathbf{L}(\mathbf{m})$ & $\mathbf{H}_{\mathbf{I}}(\mathbf{m})$ & $\mathbf{H}_{\mathbf{T}}(\mathbf{m})$ & Wave Attenuation & $\mathbf{V}(\mathbf{m} / \mathbf{s})$ \\
\hline 1 & 1.5 & 0.1 & 0.007 & 0.93 & 0.27 \\
\hline 1 & 1.5 & 0.14 & 0.008 & 0.94 & 0.3 \\
\hline 1 & 1.5 & 0.18 & 0.01 & 0.94 & 0.32 \\
\hline 1 & 1.5 & 0.22 & 0.012 & 0.94 & 0.33 \\
\hline 2 & 1.5 & 0.1 & 0.055 & 0.45 & 0.91 \\
\hline 2 & 1.5 & 0.14 & 0.058 & 0.58 & 1.05 \\
\hline 2 & 1.5 & 0.18 & 0.06 & 0.66 & 1.20 \\
\hline 2 & 1.5 & 0.22 & 0.062 & 0.71 & 1.46 \\
\hline 3 & 1.5 & 0.1 & 0.01 & 0.9 & 0.92 \\
\hline 3 & 1.5 & 0.14 & 0.016 & 0.88 & 1.17 \\
\hline 3 & 1.5 & 0.18 & 0.018 & 0.9 & 1.25 \\
\hline 3 & 1.5 & 0.22 & 0.02 & 0.9 & 1.34 \\
\hline
\end{tabular}

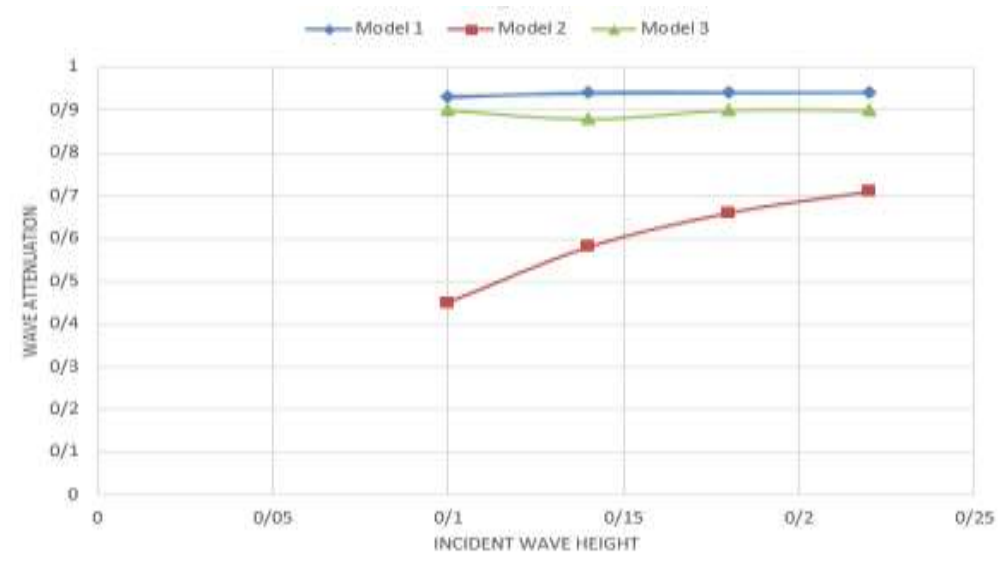

Fig. 11: The numerical simulation results of wave attenuation (Wave length $1.5 \mathrm{~m}$ ).

\section{Conclusion}

The performance of offshore stationary OWC device as a wave energy convertor and a floating breakwater have been numerically investigated. In order to do that, 3 types of OWC designed with different dimensions have been modeled and impacts of increasing the incoming wave height was analyzed.

After some tests by a comparison between model 1 and 2, it was obvious that model 1 reflected wave energy more than model 2 and it attenuated about 90 percent of incident wave height but less energy can be extracted by model 1 compared to model 2 and 3.

Model 2 let the waves pass the OWC but it extracts more wave energy compered to model 1 and the water surface level inside of the OWC fluctuated more than model 1.

Finally, model 3 cover the deficiency of both models in this model the amount of wave attenuation was in good rang and because the length of front wall is shorter than back wall, it lets wave enter the chamber but back wall does not let it pass the OWC so the water surface level fluctuates more in the chamber and it increases the trapped air inside the chamber and the air pressure increases power generation by turbines. Therefore, as the back wall of the OWC increases, more energy is absorbed so model 3 shows the better performance compared to the two other models.

This study shows OWC devices absorb wave energy and convert to other form of energy also they can be used as a floating breakwaters therefore using OWC devices are more Cost-effective compare to other form of wave energy converters and breakwaters. 


\section{References}

[1] D. V. Evans, "Oscillating water column wave energy convertors," J. IMA Journal of Applied Mathematics, vol. 22, pp. 423-433, 1978.

[2] D. V. Evans, "Wave power absorption by systems of oscillating surface pressure distributions," J. Journal of Fluid Mechanics, vol. 114, pp. 481-499, 1982.

[3] K. Thiruvenkatasamy, S. Neelamani and M. Sato, "Nonbreaking wave forces on multiresonant oscillating water column wave power caisson breakwater," J. Journal of Waterway, Port, Coastal and Ocean engineering, vol. 131, no. 2, pp. 77-84, 2005.

[4] C. Josset and A. H. Clement, "A time-domain numerical simulator for oscillating water column wave power plants," Renewable Energy, vol. 32, no. 8, pp. 1379-1402, 2007.

[5] J. Henriques, A. Falcao, R. Gomes, L. Gato, "L.M.C. Latching Control of an Oscillating Water Column Spar-Buoy Wave Energy Converter in Regular Waves," J. Journal of Offshore Mechanics and Arctic Engineering, vol. 135, no. 2, 2013.

[6] A. McKinley, P. Lamont-Kane, A. Henry, M. Folley, J. Nicholson, T. Whittaker, B. Elsaesser, "Preliminary development of a Pseudo-3D testing technique for the prediction of extreme loads on an oscillating wave surge converter," in 3rd Asian wave and tidal energy conference, Singapore, vol. 2 , 2016.

[7] A. Elhanafi, "Prediction of regular wave load on a fixed offshore oscillating water column-wave energy converter using CFD," J. Journal of Ocean Engineering and Science, vol. 1, no. 4, pp. 268-283, 2016.

[8] A. Elhanafi, G. Macfarlane, A. Fleming, Z. Leong, "Experimental and numerical measurements of wave forces on a 3D offshore stationary OWC wave energy converter," J. Journal of Ocean Engineering, vol. 144, pp. 98-117, 2017.

[9] P. Lubin, S. Vincent, S. Abadie, J. Caltagirone, "Three-dimensional Large Eddy Simulation of air entrainment under plunging breaking waves," J. Coastal Engineering, vol. 53, no. 8, pp. 631-655. 2006.

[10] R. G. Dean, R. A. Dalrymple, "Water wave mechanics for engineers and scientists," in World Scientific, 3rd Ed., USA, 1991.

[11] E. Zauderer, "Partial differential equations of applied mathematics," in Wiley (Interscience), 3rd Ed., 2011.

[12] M. Abbaspour, M. H. Ghodsi, "Design of a new scheme to indicate the domain of applicability of near singular approach in 2D BEM," Engineering Analysis with Boundary Elements, vol. 35, no. 1, pp. 129-139, 2011.

[13] M. H. Ghodsi, "Free surface modeling in OWC chamber with parabolic side walls using 3D BEM," AIP Conference Proceedings, pp. 1648, 2015. 